\title{
Retraction With Face Saving: modelling conversational interaction through dynamic hypermedia
}

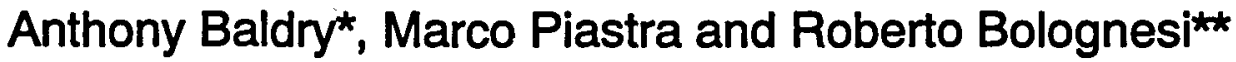

\author{
* University of Pavia, Italy \\ * CARES (Research Institute), Pavia, Italy
}

\section{Abstract}

This paper describes RWFS (Retraction With Face Saving), a hypermedia application which models an interview between a lawyer and his client - a lorry driver - facing court charges of reckless driving. At one level RWFS takes the form of a sophisticated game in which different outcomes to the interview are possible according to the learner's degree of skill. At another level, RWFS is designed to encourage the language learner's awareness and understanding of the pragmatic features of conversation. RWFS runs on HyperContext, a hybrid hypertextlexpert system developed in Pavia by two of the authors, Marco Piastra and Roberto Bolognesi, and which supports dynamic hypermedia units. HyperContext's dynamic linking capacity plays a vital role in simulating significant conversational features such as the conditioning of a current move in the conversation by information acquired much earlier in the course of the interview. In this connection, the paper discusses the contribution of RMCI (Re-usable Model of Conversational Interaction), a re-usable application-independent applied model of interaction on which the game is based, and which links a tactical level (the conversation) to a metalevel which provides a moveby-move commentary on interactional theory. In its turn, RMCr's metalevel is linked to a strategic level which interprets the structure of the conversation in terms of a pyramid-like hierarchy of increasingly abstract theoretical concepts.

\section{Introduction}

Assume a lawyer is interviewing a client. For the participants in the interview, the interaction will probably be seen in its simplest form, namely as achieving certain goals and overcoming obstacles that would otherwise prevent these goals from being achieved. For an outside observer, examining the conversation will be a more complex process that takes into account the interactants' behaviour, their social status, and knowledge and perception of each other, as well as their perception of what it is possible and appropriate to say and do at any point in the conversation. Although many excellent theoretical models exist that interpret these characteristics (see, for instance, Sinclair and Coulthard, 1975; Coulthard, 1977; De 
Beaugrande and Dressler, 1981), an application-independent applied model of interaction that can be used in a variety of computer applications to encourage a student's awareness and understanding of the pragmatic features of conversational encounters still needs to be developed. Since its educational success depends on its potential to interpret the work being done in a conversational encounter, such an application must contain a metalevel that systematically comments on the tactical level, i.e. what is actually happening in the conversation. In its turn, the tactical level must be related to a higher level, the strategic level which characterizes goals, constraints, identities and other ingredients in the encounter in terms of specific theories of interaction. The resulting application will thus provide a commentary that interprets the tactics that the participants adopt in pursuit of their conversational goals, but which in its turn is linked to a more theoretical account of how the participants' tactics are achieving these goals and coping with the social and behavioural conditions placed on their attainment.

RWFS (Retraction With Face Saving) is designed to fulfil these objectives. It simulates a lawyer-client consultation in which the goal of the lawyer (LW) is to get his client, a lorry driver (LD), to rectify a misleading statement made to the police following a road accident. The description given in this paper relates to the tactics needed to complete the first stage in the game, namely the LW gets the LD to agree to retract his statement without humiliating him in the process. As the introductory case notes (Figures 1 and 2) show, RWFS implements the strategic level in terms of intentionality and acceptability (De Beaugrande and Dressler, 1981; Searle, 1983; Searle et al., 1992).

The simulation takes the form of an adventure game with rules that must be respected. In order successfully to negotiate the individual stages in the LW's conversational plan, the student must respect the conditions placed on the LW in the conversation by the LD's personality, by the social conventions that operate in a lawyer-client consultation, and more generally by the structure of conversation itself. The student is required to follow a tactical progression that keeps the LD's willingness to participate in the dialogue alive. The tactical progression consists of a series of moves made by the LW (a multiple-choice option selected by the student). The LW has to adopt tactics that fall within the range of (psychological) acceptability to the client, since each move causes the LD's degree of 'one-up/one-down' domination (Watzlawick et al., 1967), co-operation and trust (Grice, 1975) to be modified.

As Figure 3 indicates, there is no single winning path through the materials. In order to proceed to the second stage in the game, a student must achieve the interactional goal (the LD's ADMIT), respecting social conventions and norms, in particular the need to preserve the LD's face. Visual and textual clues relating to the LD's reactions (and hence to the student's skill in handling the interview) are given as the game proceeds. With the exception of three 'fatal' moves, marked Exit in Figure 3, no single move will cause the LD to seek another lawyer, and provided the tactics fall within a range of acceptability to the $L D$, the first stage in the game can be taken to a successful conclusion. There are many possible outcomes, each associated with different conversational and psychological states. Some of these outcomes mean that the student wins the game; others that he or she has failed.

Five basic problems face the researchers wishing to model a simulated interview which, though implemented as an adventure game, still remains sophisticated enough to act as an introduction to conversational theory for students of linguistics:

(i) scripting the simulated interview in such a way as to provide for convincing alternative conversational states during the proceedings, as well as a convincing outcome; 


\section{Drawing up a conversational strategy}

You are a lawyer (LW). A few days ago you were contacted by a lorry driver (LD) involved in a road accident. While you are waiting for him to arrive, you decide your conversational strategy and tactics. You suspect that your client will lose his licence, at the very least. You also realize that the statement he has made to the police is inaccurate and must be rectified. By all accounts, he is clearly an irascible person so you must tread carefully. You must do four things in this interview:

(1) make sure your client tells you the truth about the accident;

(2) get your client to withdraw from his current position;

(3) tell your client the truth about his licence, if he asks you;

(4) show the client various documents at the right time and in an appropriate order: these include police reports, medical examinations, newspaper reports, statements and documents relating to previous cases.

\section{Dividing up the conversation into sub-goals and sub-sub-goals}

In order to proceed to the second sub-goal you must successfully complete the first subgoal: ADMIT.

This requires the LD to recognize that certain passages in his statement are not in his favour. This is divided into four sub-sub-goals. Each establishes a desired state whose achievement requires you to adopt specific tactics. Your plan is to establish:

(1) a starting point in the discussion: by selecting which documents to leave in full view on the table, you decide to reveal how much you know about the case; by setting the declared (as opposed to real) shared information, you also implicitly establish your subsequent tactics by choosing one of four initial conditions: Hide newspaper; Hide statement; Hide both; Hide nothing. Your choice of initial condition will influence the dialogue, but you should attempt to manipulate these conditions to your advantage as the game goes on.

(2) recognition of stress by the $L D$ : the LD's actions (and in particular his statement) were the result of the stressful circumstances at the time; you use the newspaper article and/or the information it contains to achieve this recognition; decide your tactics carefully.

(3) an acceptable pre-retraction state: namely the idea that because the statement was given under stressful circumstances, it contains errors and ambiguities; you get the LD to agree that a misleading statement is understandable but regrettable; you use his statement - but what are your tactics in using it?

(4) an admission that retraction is necessary: having established that (a) the statement was given under stressful circumstances - almost under duress - and that (b) there are inaccurate and misleading details in the statement, your tactics now require you to get the LD to agree to retract; you decide to play your ace, showing the LD the confidential report and/or the information it contains in an appropriate way - how in practice will you play this ace?

The phone rings and your receptionist tells you that your client is on his way to your office

Figure 1: Case notes: the lawyer's conversational plan 


\section{Coping with the lorry driver's conversational plan}

It will be hard to stop the client from trying to impose his own conversational plan on you. He wants to find out whether he will lose his licence. He is not an easy client, and there will be times when he will be dominating, uncooperative and highly suspicious - the very opposite of what you want. The trick is to make sure these attitudes are kept within an appropriate range so that you keep the conversation going, steering it towards your own conversational goals. You cannot simply ignore your client or simply lay down the law with him; any conversation is a process of negotiation. In this chess-like game, you need to work out tactics that take into account the possible responses that your client will make both immediately and at a later stage in the conversation as the result of your current move.

\section{Anticipating the lorry driver's probable reactions}

Good and bad moves are defined in terms of their acceptability or otherwise to your client. The range of acceptability is defined numerically in terms of three parameters: dominance, co-operation, trust. These function independently of each other, and each move you make will cause the current state of each parameter to go up or down or stay the same. Ideally, you want dominance to decrease, co-operation and trust to increase. The feedback from the client - his reaction to your move - will, of course, influence and become part of the subsequent conversation. Use your common sense and knowledge of the previous, current and probable subsequent states in the conversation to keep the conversation going.

Figure 2: Case notes: the lorry driver's conversational plan and likely reactions

(ii) getting the computer to simulate the behaviour of one of the parties in a two-party conversation, and that party's reactions to the conversational tactics and strategies adopted by the other party, played in a chess-like game, by the student;

(iii) capturing the dynamic nature of conversation, a process in which the decisions made by the parties place constraints on their subsequent conversation;

(iv) defining mechanisms by which to associate an amusing game to rather more serious theories of conversational interaction;

(v) creating a re-usable model of conversational interaction that can be used as a basis for subsequent related applications. Because of the complexity of these issues, and restrictions on space, most of what follows relates to the tactical level, and only a brief reference is made to the metalevel and the strategic level.

\section{RWFS and the definition of the tactical level}

\section{Scripting and the problem of modelling dlalogue pathways In hypertext}

As mentioned above, the first problem relates to the scripting of an interview in such a way as to provide for convincing alternative conversational states during the proceedings, as well as a convincing outcome. Figure 3 gives an overview of the tactical level (limited to the first subgoal) which shows that the set of possible hypertext pathways coincides with an equivalent set of dialogue pathways: the user impersonates one of the two interactants and at each step 
makes a choice as to the next move to make along the dialogue pathway. The nodes are divided into four sections corresponding to each of the four sub-sub-goals (hence nodes $\mathrm{LW}$ $1.1,2.1,3.1,4.1$ etc.).

\section{Simulating the other party's behavlour}

RWFS relies on a working memory, a means by which the history of the moves along the dialogue pathway and the current state of the exchange between the two interactants are recorded. As indicated in the second paragraph of Figure 2, simulating the other party's behaviour is achieved in terms of three parameters - co-operation, dominance and trust - that change the state of the working memory in such a way that a visual and textual response can be given to the student. These parameters are expressed as conditions associated with each step taken along the dialogue pathway. In Figure 3 they are marked respectively as C, D, T; the symbols + and - represent changes in their values associated with a given choice of move in the dialogue. In other words, the LD's linguistic-pragmatic interpretation of the LW's moves is associated to each step in the dialogue. But this association is carried out dynamically.

In order to understand the concept of dynamic management of dialogue, a key concept in RWFS is the following theorem:

a conversational state $=$ the current hypertext node + the state of the working memory

When an application is authored in HyperContext, rules are associated by the author to every possible hypertext move. These rules provide for the updating of the working memory. The history of the student's passage along a dialogue pathway recorded in the working memory is in reality a synthesis of the major events which may affect the entire history (previous, current and subsequent) of the dialogue. The working memory update is not directly perceived by the user, but its effects are constantly present. The modelling of the conversational and psychological state of the other party in RWFS, i.e. the LD, is managed through the working memory. Hence the presentation of the LD with an angry or happy face depends entirely on the state of the working memory. Similarly, as Figure 3 shows, the initial conditions (Hide newspaper, etc.) are recorded in the working memory ( $+\mathrm{HN}$ or $+\mathrm{HS}$, etc.). A question mark indicates a consistency check. In other words, according to the way the game is played, the state of the working memory will be checked against the rules associated with a particular node.

\section{Conversational exchanges and moves}

The best-known theoretical model of conversational interaction is the rank-and-scale model associated with Coulthard, Sinclair and their colleagues (Sinclair and Coulthard, 1975; Coulthard, 1977, 1981, 1992) which provides for five levels of analysis, and in which each higher level consists uniquely of elements taken from the level immediately below. Thus an interactional encounter contains one or more transactions, a transaction one or more exchanges, an exchange one or more moves, a move one or more acts. The tactical level of RWFS is systematically and exclusively defined in terms of exchanges and the moves they consist of. A hypertext node in the tactical level corresponds to a move in the rank-and-scale model. As Figure 3 shows, the symbol $\square$ represents an LW node, and hence an LW move, while the symbol $\square$, represents an LD node, and hence an LD move. All the exchanges in the current version of RWFS are made up of an LW move followed by an LD move. 


\section{The concept of different conversational states despite apparently identical circumstances}

Like other hypertext units, the nodes in RWFS contain text and images. These are static nodes: the texts, the images and their composition do not change as the student progresses along a dialogue pathway. The nodes in the tactical level are not accessible in a completely free way: the sequences linking nodes at the tactical level (see Figure 3) are the only sequences which form a coherent dialogue pathway. The coherence of the pathways at the tactical level is guaranteed by the arrangement of the hypertext links and by the consistency rules affecting these links. The consistency rules are established by the author (in the same way as specified above under Simulating the other party's behaviour, namely by associating rules to every possible hypertext move). In most nodes, the user can choose between different moves. The consistency rules make the links dynamic, thus governing access between the nodes. To simplify the work of the author, different dialogue pathways may share one or more hypertext nodes. This means that there is no need to author each dialogue pathway separately. As mentioned above, the way in which a node is accessed determines the state of the conversation in that node and the possible continuation of the conversation subsequently. Thus the user may find himself or herself in apparently similar circumstances (i.e. the presentation of the same text and images) and yet be faced with conversational states that are entirely different. Take, for example, the node marked LW 2.1 (Show the article). This may be accessed from:

(a) node LD 1.1.2 (1);

(b) node LD 3.1.1 (1)

(c) node LD 3.1 .1 (2).

In contrast to case (a), access from (b) or (c) entails a reversal of the order in which the subsub-goals are achieved, with sub-sub-goal 3 preceding sub-sub-goal 2 . In other words, accessing the same node through two different pathways means having two different states in the working memory.

\section{Dynamic interpretation}

The previous section describes the tactical level. This provides the game component. But we need something that associates an amusing game to rather more serious theories of conversational interaction and that views a conversation as a dynamic, on-going process in which the decisions made by the parties will place constraints on their subsequent conversation. How does a researcher keen to create an applied model of interaction go about this particular task?

\section{The need for a connection between the game level and the theoretical models}

Clearly there is a need to link up the tactical level to the strategic level in a context-dependent way in order to establish what specific aspects of conversational theory are being illustrated at a given point in a conversation. But it is also necessary to organize the strategic level in a noncontext-dependent way such that the student can browse through the materials and gain a general overview of the conversational theory being presented. How can these conflicting principles be resolved? The strategic level itself is organized as a pyramid in which the characterization of, for example, sub-sub-goals is the lowest level, linked to a higher set of subgoals, in turn linked to a still higher level of goals, and so on until the top of the pyramid is 


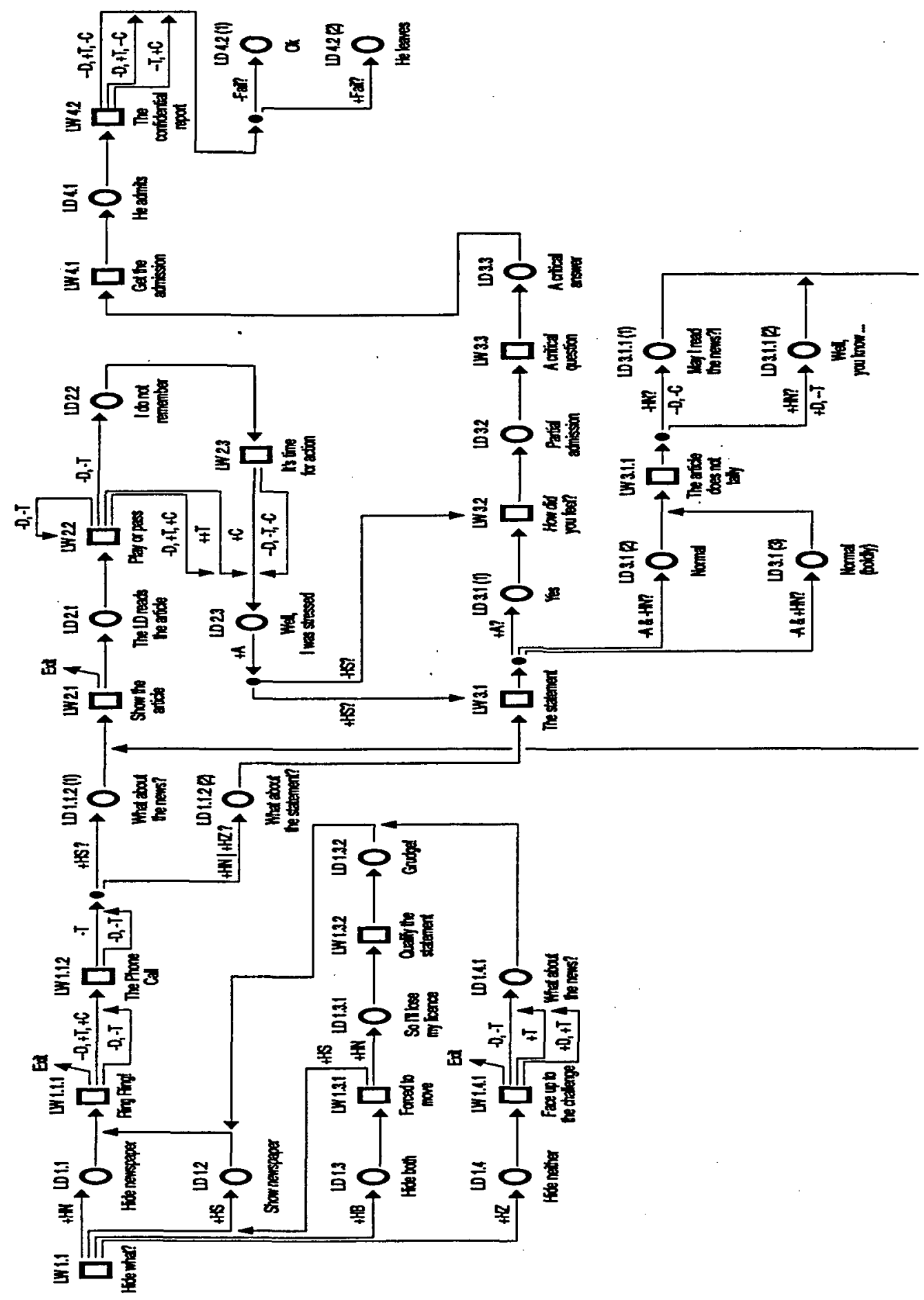

Figure 3: Possible pathways: a map of the four sub-sub-goals 
reached. Students can browse through the pyramid at will. However, access to the strategic level can be gained only from the tactical level and this link is made to specific points in the strategic level, the point of access changing as the game proceeds. Access to a specific strategic level node is achieved by clicking on a highlighted keyword in the metalevel commentary associated to a particular move. It may well be that a different choice made at the same node will require access to a different point in the strategic level. This is one reason why the link between strategic and tactical levels is made through a separate level: the metalevel.

\section{Providing a dynamic interpretation environment}

The link between a move and a strategic level node is achieved by associating interpretive hypertext nodes (the metalevel commentary) to the dialogue pathway nodes (the tactical level). This association also has to be dynamic to work properly. Access to the interpretive nodes depends on the state of the conversation, i.e. on the node reached in the dialogue pathway and the state of the working memory. The interpretive nodes explain, in a context-dependent way, the theoretical significance of a conversational state vis-à-vis a possible model of dialogue interpretation. Because of the complexity of authoring such a representation of interaction, where a change at one level has consequences at the other levels, it was decided in the early (prototype) design stages to consider a single feature as primary at all three levels, from a theoretical standpoint. The choice fell on the exchange, as this ensures the necessary focus on the relationships between the participants in a conversational encounter. Thus, although the tactical level is organized in terms of moves and exchanges, the links with other levels are achieved in terms of exchanges. Other interactional features, such as the individual moves contemplated by each participant, their speech acts, their decisions as regards turn-taking and next-speaker selection, changes in mood and body language, are considered essential but subordinate. With its focus on the notion of exchange, the proposed applied model of interaction takes changes in the state of the shared information, the mutual recognition of the roles and identities of participants and their mutual perception of other participants' moods into account. More significantly, the model captures the constraints imposed on subsequent exchanges by the previous history of the conversation.

\section{Connecting the tactical level to the strategic level through the metalevel}

In just the same way as the dialogue pathway nodes collectively make up the tactical level, so the interpretive nodes collectively make up the metalevel. The strategic level consists of hierarchically organized hypertext nodes in which the author describes and interprets the sequences in a dialogue pathway according to the chosen theoretical model. The strategic level, moreover, has the goal of justifying the alternative sequences of moves and exchanges in the dialogue pathways and illustrating the restrictions on the possible continuation introduced by the choices made. The interpretive nodes (i.e. the metalevel) serve to correlate each accessible conversational state with the theoretical explanations contained in the strategic level.

\section{Model re-usability}

\section{Even authors may well get lost In hyperspace}

The construction of a hypermedia unit like RWFS can require the author to create hundreds, possibly thousands, of different nodes. The organization into three distinct levels helps the author to dominate the complexity of the application. Within certain limits the three levels can 
be subdivided and managed separately. Each level can be further organized (e.g. a flow chart containing possible dialogue pathways for the tactical level, a hierarchical pyramid structure for the strategic level). Hence the conception of RWFS as being based on a more abstract application-independent applied model of interaction denominated RMCI (Re-usable Model of Conversational Interaction).

\section{The RWFS paradigm can be re-used}

Thanks to the RMCI, the material contained in RWFS can be re-used in other units. The metalevel nodes act in such a way as to separate the tactical level and the strategic level, thus enhancing the possibility of re-use. The tactical level may be interpreted in terms of one or more theoretical interpretive models, and vice versa the strategic level can be linked to different tactical levels. Thus, for example, although the current model of interaction is heavily geared towards an understanding of the concept of exchange, there is no reason why the tactical level could not be linked to an entirely different theoretical interpretation. Indeed, the reversal in the order in which sub-goals are tackled (see above under Conversational exchanges and moods) indicates RMCI's capacity to manage whole chains of exchanges as a separate block. In other words, RMCI already contains the wherewithal to handle conversational units higher than the exchange. It may well turn out to be the case that RMCI can be used in conjunction with end-applications that require, for example, the incorporation of Coulthard and Sinclair's concept of transaction (Sinclair and Coulthard, 1975; Coulthard, 1992).

\section{RWFS, RMCl and HyperContext}

Although both RWFS and RMCI represent a simplified interpretation of interaction, many of their features cannot be captured with the current generation of commercially available hypertext tools. The design criteria of HyperContext, a hybrid hypertext/expert system, are such as to take into account the complex work of tracking and updating changes that occur at all three levels in the applied model of interaction. This is in itself a complex game of mutually conditioning and interlinked actions. Since the description of RMCI and HyperContext in this paper is necessarily reductive, readers are referred to the technical report on HyperContext (Piastra and Bolognesi; 1993). It is hoped to publish a fuller account of HyperContext's potential value in applied linguistics at a later date. This will indicate how important it is to develop re-usable applications that represent an intermediate level between an authoring system and an end-user application.

\section{Conclusion}

The description given above is technical, but shows how dynamic hypermedia can present conversation in a holistic way and from many different viewpoints - an essential requirement if conversational theory is to be tackled properly. In this respect, the goal of RWFS is to encourage students to acquire the following skills:

- relating the surface structure of the conversation (the tactical level) to the underlying belief and behavioural systems (accounted for in the strategic level);

- the constraints - social and linguistic - placed on the interactants;

- the changing relationship between the interactants and the changes in the roles that they play; 
- changes in the shared information as the conversation proceeds;

- turn-taking and next-speaker selection;

- other factors affecting the course of the conversation such as the need to preserve face.

We may view these skills as relating, at least in part, to a student's metapragmatic competence - taken here as being a student's ability to describe what is going on in a conversation and relating it to theoretical models of interaction. Such skills can be secured only with the development of specialized techniques and frameworks in which they can be practised. A dynamic hypermedia model of at least some aspects of conversation seems to be effective. In pursuing the goal of creating suitable computer-based techniques and frameworks, research is needed in specialized fields, like the one proposed here, which entail the modelling of the interplay of many linguistic phenomena from many different standpoints. This project thus represents a specialist approach to multimedia in which the relationship of data to a critical and theoretical interpretive framework is an overriding consideration.

But will it help students? How do students react to the view of pragmatics as presented in RWFS? Will they find it as dull as ditchwater or will it help them to pin down the eel-like, multifaceted interplay of factors in conversation which are so hard to cope with? It must be stressed that RWFS is an alpha-prototype application, based on an alpha-prototype applied linguistics model of interaction which in turn runs on an alpha-prototype system whose educational validity is in the alpha-stage of evaluation. The application has all the weaknesses of a prototype, most seriously that it does not use authentic interactional data. It is also a heavily simplified model of face-to-face interaction, which takes the form of an interview made up of a series of exchanges, each consisting rigidly of a client move followed by a lawyer move. The work of experimentation and validation of the application may well indicate a need for changes to be made. But, in focusing on pragmatic principles RWFS does make students more aware of the nature of interviews as a social activity; it does relate interviews to other types of conversational interaction; it does focus on the asymmetries that occur in interviews, and it does highlight the consequences when a particular exchange sequence is adopted. It is a start (but only a start) to the complex work of realizing the potential of computers to relate the study of language more fully to social behaviour and cross-cultural differences.

\section{Acknowledgements}

The authors wish to thank Lavinia Merlini Barbaresi, University of Pisa, and Carol Taylor Torsello and Franco Crevatin, University of Trieste, for their help during the development of RWFS. Part of the applied research and experimentation with RWFS is being undertaken within the Apprendimento Linguistico in Ambiente Ipermediale project, MURST 40\%.

\section{References}

Coulthard, R.M.(1977), An Introduction to Discourse Analysis, London, Longman.

Coulthard, R.M. (1981), Studies in Discourse Analysis, London, Routledge.

Coulthard, R.M. (1992), Advances in Discourse Analysis, London, Routledge.

De Beaugrande, R. and Dressler, W. (1981) Introduction to Text Linguistics, London, Longman.

Grice, P. (1975) 'Logic and conversation' in Cole, P. and Morgan, J. (eds) Syntax and Semantics III: Speech Acts, New York, USA, Academic Press, pp. 41-58. 
Piastra, M. and Bolognesi, R. (1993), A Brief Description of HyperContext, Technical Report 001-93, CARES, Pavia, Italy.

Searle, J.R. (1983), Intentionality. An Essay in the Philosophy of Mind, Cambridge University Press.

Searle, J.R. et al. (1992), (On) Searle on Conversation, Philadelphia, USA, John Benjamin.

Sinclair, J.M. and Coulthard, R.M. (1975), Towards an Analysis of Discourse, Oxford University Press.

Watzlawick, P., Helmick, B.J. and Ackson, D.D. (1967), Pragmatics of Human Communication. A Study of Interactional Patterns, Pathologies and Paradoxes, New York, USA, Norton. 